\title{
Inverse scattering for time-varying one-dimensional layered media: algorithms and applications
}

\author{
Andrew E Yagle $\dagger$ \\ Department of Electrical Engineering and Computer Science, The University of Michigan, \\ Ann Arbor, MI 48109-2122, USA \\ Received 13 February 1995, in final form 1 April 1997
}

\begin{abstract}
We formulate and present algorithms and applications for the inverse scattering problem for a one-dimensional layered medium whose reflection coefficients vary with time. We show that given the reflection responses to impulsive plane waves incident on the medium at different times, this problem can be solved either by solving a series of nested non-Toeplitz systems of equations, or by propagating a set of coupled layer-stripping algorithms with reflection coefficients varying in time. All multiple scattering effects are included here. We show that our results reduce to previous results in the special case of time-invariant media. Applications include the two-dimensional (2D) inverse resistivity problem of reconstructing the 2D resistivity $\rho(x, y)$ of a 2D medium from surface measurements, and the reconstruction of rapidly curing layered media. We also present a time-varying discrete Miura transform linking the time-varying discrete wavesystem inverse scattering problem to a time-varying discrete Schrödinger equation, and a new important feasibility condition on the reflection responses, which seems to be new in the context of the $2 \mathrm{D}$ inverse resistivity problem. A numerical example is provided.
\end{abstract}

\section{Introduction}

The inverse scattering problem for a one-dimensional (1D) laterally homogeneous layered medium with time-invariant (i.e. constant) layer impedances has been studied for many years. For a good review see [1]. By formulating this problem in terms of downgoing and upgoing waves which are reflected into each other at interfaces, this problem can be related to fast algorithms in digital signal processing, such as the Levinson and Schur algorithms, and to the nested Toeplitz systems of equations these fast algorithms solve [2]. In the continuous limit of the discrete problem, the nested Toeplitz systems of equations become the Krein integral equation, and the Levinson and Schur algorithms become layer stripping algorithms [3]. Since implementation of layer-stripping algorithms requires discretization, it makes sense to discretize them to the Levinson and Schur algorithms, since these algorithms are numerically stable in the presence of noise, while other discretizations are usually unstable in noise.

Schur and Levinson-type layer-stripping algorithms do diverge sometimes, when the two-sided free-surface impulse reflection response is not positive definite. This positive definiteness is not merely a prerequisite for stability of the algorithms, but a feasibility condition for the inverse problem data [7]. Thus considering the discrete problem directly leads not only to stable layer-stripping algorithms, but also to results about the problem itself. The continuous problem can also be formulated in terms of a Schrödinger equation

† E-mail address: aey@eecs.umich.edu 
[4]; this formulation is related to the two-component wavesystem formulation using the Miura transform [3].

If the layer impedances are allowed to vary with time, the problem becomes more complicated, since the reflection coefficients vary with time and the multiple scattering occurs off of different-valued coefficients at different times. There has been no generalization of the results of $[2,3]$ to the time-varying case. The time-varying case has several interesting applications which are summarized in section 5. For example, a layered composite with rapidly curing adhesive layers bonding the layers on either side can have its curing progress determined by using a non-destructive evaluation. Also, the $2 \mathrm{D}$ inverse resistivity problem can be reformulated as a time-varying $1 \mathrm{D}$ inverse scattering problem. Details are given in section 5 .

This paper performs the generalization of the elegant formulation of $[2,3]$ to the timevarying case. We show that given the reflection responses to impulses incident on the medium at different times, time-varying reflection coefficients can all be reconstructed for all times. This can be done by either using a set of coupled layer-stripping algorithms with time-varying reflection coefficients, or by solving a set of nested non-Toeplitz systems of equations. The non-Toeplitz nature of these systems of equations is a direct consequence of the time-varying reflection coefficients-we show that for a time-invariant medium our results reduce immediately to the more familiar results of $[2,3]$. Continuous versions of our results can be found immediately from the limit of the discrete results. However, we point out in section 5.2 that the continuous time-varying problem is not properly defined by just its equation; some explanation of the relation between times at which the medium undergoes its changes and times at which waves interact with the medium is required.

We also present an important new application of our results to the 2D inverse resistivity problem of reconstructing a 2D varying resistivity $\rho(x, y)$ (or conductivity $\sigma(x, y)=$ $1 / \rho(x, y))$ from surface measurements of the voltage response to a current source. This problem has attracted great interest lately [5,6]. However, our results differ from most impedance tomography results [13] in that all multiple scattering effects are included-the Born approximation is not required. This can be significant when $\rho(x, y)$ is much larger or smaller in some regions than its background value. Details of the connection between the 2D impedance tomography problem and the $1 \mathrm{D}$ time-varying inverse scattering problem are presented in [14]; we summarize them here for the half-space problem.

We also present a new time-varying Miura transform that relates the time-varying wavesystem problem to a time-varying Schrödinger equation inverse potential problem. We also derive a new feasibility condition on the reflection response of a time-varying medium; this result is directly applicable to the $2 \mathrm{D}$ inverse resistivity problem, and seems to be new in that context.

This paper is organized as follows. The basic problem is formulated in section 2 and solved using layer-stripping-type algorithms in section 3 and using nested non-Toeplitz systems of equations in section 4 . The new feasibility condition is also derived in section 4 . Applications, including the 2D inverse resistivity problem, are presented in section 5 (these come later since they must be adapted to the formulation presented in section 2). The time-varying discrete Miura transform is also presented in section 5. Section 6 provides an illustrative numerical example which demonstrates our results. Section 7 concludes with a summary and suggestions for further research. 


\section{Problem formulation}

The problem we address is as follows. A discrete 1D layered medium consists of a stack of laterally homogeneous layers. The impedance of the $n$th layer is $Z_{n}(i)=\rho_{n}(i) c_{n}$ where $\rho_{n}(i)$ and $c_{n}$ are the density and wavespeed. The thickness of the $n$th layer is $c_{n} / 2$ so that the two-way travel time through each layer is unity. This is the usual Goupillaud medium assumption [1-4]. If the $1 / c_{n}$ are integer multiples of a slowness $1 / c_{0}$, then the medium can be regarded as a stack of layers of unit thickness with wavespeed $2 c_{0}$ throughout (some layers will be identical to those above and below them). In the sequel we assume this and scale $c_{0}=1$. Except for the time variation of the density $\rho_{n}(i)$, this is the same formulation as the one used in [1-4], which has been successfully applied to reflection seismology $[1,4]$ and transmission lines [2].

\subsection{Wave propagation in a time-varying medium}

Wave propagation in the medium is described by the equations

$$
\begin{aligned}
& {\left[\begin{array}{l}
d_{n}(i, j) \\
u_{n}(i, j)
\end{array}\right]=\frac{1}{t_{n}(i)}\left[\begin{array}{cc}
1 & -k_{n}(i) \\
-k_{n}(i) & 1
\end{array}\right]\left[\begin{array}{l}
d_{n-1}(i-1 / 2, j) \\
u_{n-1}(i+1 / 2, j)
\end{array}\right]} \\
& t_{n}(i)=\sqrt{1-k_{n}^{2}(i)} \quad k_{n}(i)=\frac{Z_{n}(i)-Z_{n+1}(i)}{Z_{n}(i)+Z_{n+1}(i)} .
\end{aligned}
$$

Here $d_{n}(i, j)$ and $u_{n}(i, j)$ are downgoing and upgoing waves just below the $n$th interface (which is between the $n$th and $(n+1)$ th layers) at time $i$, due to an impulse which is at the top (0th interface) of the medium at time $j . k_{n}(i)$ is the reflection coefficient at the $n$th interface at time $i$. Without a loss of generality, we assume that $k_{n}(i)$ is a piecewise constant, changing just before half-integer times. Note that the wave interactions described in (2.1) all occur at time $i$. This does not impose any physical restriction, since waves are reflected off the $n$th interface only at times $i$ for which $n / 2+i+j$ is an integer. The details of the derivation of (2.1) in the time-invariant case $k_{n}(i)=k_{n}$ are presented in [1-4] and will not be repeated here. It is worth noting that since waves require 0.5 time units to pass through a layer, waves will be incident on the $n$th interface at half-integer times if $n$ is odd. Making $k_{n}$ time-varying does not alter any significant aspects of the derivations in [1-4], since the wave interactions that occur in the derivations all occur at a single time $i$. The physical interpretation of (2.1) as downgoing waves being scattered into upgoing waves (and vice versa) by reflection coefficients should be apparent.

We also assume without a loss of generality that in physical experiments the layer and interface index $n$ and time indices $i$ and $j$ are all non-negative, i.e. no experiment starts at a negative time $j$. Note that the actual time origin can be set arbitrarily long ago. We also define $k_{n}(-i)=k_{n}(i)$, i.e. $k_{n}(i)$ is symmetric about the time $i=0$. Since $j \geqslant 0$, the first wave interaction at the $n$th interface occurs at time $i=n / 2+j \geqslant n / 2$, so this assumption has no effect on the physical problem. It does make the form of the matrix Green's function $H^{n}(i, k)$ in section 4.2 simpler.

These are the same equations as in [1-4] except that reflection coefficients $k_{n}(i)$ are now allowed to be time varying. Note that since the waves are energy normalized (see [1]) the varying of layer density while the waves are propagating between layers does not affect their amplitude. Also recall that we assume that the wavespeed does not vary with time. 


\subsection{Boundary conditions}

The boundary conditions for (2.1) are

$$
d_{0}(i, j)=\delta(i-j)+r_{i, j} \quad u_{0}(i, j)=r_{i, j} \quad d_{N}(i, j)=x_{i, j} \quad u_{N}(i, j)=0 .
$$

Here $r_{i, j}$ is the free-surface impulse reflection response at time $i$ to an impulse which is at the top of the medium at time $j . x_{i, j}$ is the transmission response that comes out of the bottom ( $N$ th interface) of the medium. This describes an experiment in which an impulse $\delta(i-j)$ initiated at time $j$ is used to probe the medium, resulting in a reflection response $r_{i, j}$ and a transmission response $x_{i, j}$. For a time-invariant medium, we would have $r_{i, j}=r_{i-j}$ and $x_{i, j}=x_{i-j}$, since delaying the excitation by time $j$ would simply delay the response by time $j$. The free surface assumption means that the surface of the medium is assumed to be a perfect reflector, which is reasonable since in many applications there is a huge impedance mismatch between the medium and its exterior (e.g. between a solid and air).

The inverse scattering problem is to reconstruct the time-varying reflection coefficient $k_{n}(i)$ from the reflection responses $r_{i, j}$ to impulses at integer times $j \geqslant 0$. We discuss ways to obtain such data in section 5 .

\section{Solution using layer stripping}

From (2.1) and (2.2) it is physically clear that the waves $d_{n}(i, j)$ and $u_{n}(i, j)$ have the causality properties

$d_{n}(i, j)=0 \quad$ if $i<(n / 2)+j \quad u_{n}(i, j)=0 \quad$ if $i<(n / 2)+j+1$.

Using (3.1) and setting $i=n / 2+j$ in the bottom row of (2.1) gives

$$
k_{n}(n / 2+j)=u_{n-1}((n+1) / 2+j, j) / d_{n-1}((n-1) / 2+j, j)
$$

which states that the first non-zero value of the upgoing wave at depth $n-1$, which occurs at time $i=(n+1) / 2+j$, is the reflection of the first non-zero value of the downgoing wave at depth $n-1$ (which is the attenuated impulse at time $(n-1) / 2+j$ ) off the $n$th interface at time $n / 2+j$.

Now suppose that we have the free-surface impulse reflection response data $r_{i, j}$ for all integers $i, j \geqslant 0$. Then we can recursively propagate (2.1) and (3.2), initialized using (2.2), as the following layer-stripping algorithm:

$$
\begin{aligned}
& d_{0}(i, j)=\delta(i-j)+r_{i, j} \quad u_{0}(i, j)=r_{i, j} \quad i, j \geqslant 0 \\
& {\left[\begin{array}{l}
d_{n}(i, j) \\
u_{n}(i, j)
\end{array}\right]=\left[\begin{array}{cc}
1 & -k_{n}(i) \\
-k_{n}(i) & 1
\end{array}\right]\left[\begin{array}{l}
d_{n-1}\left(i-\frac{1}{2}, j\right) \\
u_{n-1}\left(i+\frac{1}{2}, j\right)
\end{array}\right]} \\
& k_{n}(n / 2+j)=u_{n-1}((n+1) / 2+j, j) / d_{n-1}((n-1) / 2+j, j) .
\end{aligned}
$$

Note the following about algorithm (3.3):

(1) since $j$ is restricted to integers, $i$ takes on half-integer values if and only if $n$ is odd;

(2) we omit $t_{n}(i)$ from (2.1) since it cancels in (3.2); this saves computation time;

(3) algorithm (3.3) must be propagated simultaneously for all values of $j$, since $k_{n}(i)$ must be computed for all $i$ and broadcast to all copies of (3.3) at each recursion;

(4) we can easily use a half-space boundary condition instead of the free surface boundary condition by changing to $d_{0}(i, j)=\delta(i-j)$;

(5) if an $N$-layer medium is to be reconstructed at $N$ different times, we require $\left\{r_{i, j}, 0 \leqslant i, j \leqslant N\right\}$ and $\mathrm{O}\left(N^{3}\right)$ multiplications and additions; 
(6) for the special case of a time-invariant medium $k_{n}(i)=k_{n}$, we only require (3.3) for a single value of $j$, so there is no coupling between several copies of (3.3), which reduces to the Schur algorithm $[2,3]$.

\section{Solution using systems of equations}

We can also solve the time-varying inverse scattering problem by solving a nested set of non-Toeplitz systems of equations, as we now show. The results of this section generalize the matrix results of $[1-4]$ to the time-varying medium case. We also obtain feasibility conditions for the reflection responses.

\subsection{Time-reversal and adjoints of medium impulse responses}

Recall that $r_{i, j}$ is the reflection response at time $i$ to an impulse which is at the surface $n=0$ at time $j$, where $i, j \geqslant 0$. From (3.1) $r_{i, j}=0$ for $i<j+1$. Now reverse time by replacing $i$ with $-i$ and $j$ with $-j$, and define $r_{i, j}$ for $i, j<0$. Since $k_{n}(i)=k_{n}(-i)$, we now argue that

$$
r_{-i,-j}=r_{j, i}
$$

To see this, consider a specific example. Fixing $j=0$, we have directly that

$$
\begin{aligned}
& r_{1,0}=k_{1}\left(\frac{1}{2}\right) \quad r_{2,0}=k_{2}(1)-k_{1}\left(\frac{1}{2}\right) k_{1}\left(\frac{3}{2}\right) \\
& r_{3,0}=k_{3}\left(\frac{3}{2}\right)+k_{1}\left(\frac{1}{2}\right) k_{1}\left(\frac{3}{2}\right) k_{1}\left(\frac{5}{2}\right)-k_{2}(1) k_{1}\left(\frac{5}{2}\right)-k_{1}\left(\frac{1}{2}\right) k_{2}(2) .
\end{aligned}
$$

Now fix $i=0$, and consider what responses would be measured at $i=0$ from experiments initiated at times $j=-1,-2,-3$ :

$r_{0,-1}=k_{1}\left(-\frac{1}{2}\right) \quad r_{0,-2}=k_{2}(-1)-k_{1}\left(-\frac{1}{2}\right) k_{1}\left(-\frac{3}{2}\right)$

$r_{0,-3}=k_{3}\left(-\frac{3}{2}\right)+k_{1}\left(-\frac{1}{2}\right) k_{1}\left(-\frac{3}{2}\right) k_{1}\left(-\frac{5}{2}\right)-k_{2}(-1) k_{1}\left(-\frac{5}{2}\right)-k_{1}\left(-\frac{1}{2}\right) k_{2}(-2)$.

Comparing (4.2) with (4.3), it is clear that the response $r_{i, 0}$ is the same as the response $r_{-0,-i}$ of a medium in which $k_{n}(i)$ has been replaced with $k_{n}(-i)$, i.e. a medium in which time has been reversed.

Some reflection shows that this principle holds in general. The response observed at a fixed time $i$, due to a series of experiments initiated at various times $j<i$, consist of all primary and multiple reflections requiring a total time of $2(j-i)+1$. The responses observed at a series of times $i>j$, due to a single experiment initiated at time $j$, consist of all primary and multiple reflections requiring a total time of $2(i-j)+1=2((-j)-(-i))+1$. The primary and multiple reflections occur at times measured backward from $i$ in the first case and forward from $j$ in the second case; this explains why the reflection coefficients must be reversed in time as well.

Now consider an experiment in which the layered medium is probed from below. That is, the boundary conditions (2.2) are changed to

$$
u_{N}(i, j)=\delta(i-j) \quad d_{N}(i, j)=r_{i, j}^{\prime} \quad u_{0}(i, j)=x_{i, j}^{\prime} \quad d_{0}(i, j)=x_{i, j}^{\prime} .
$$

Equations (4.4) describe an experiment in which a probing impulse $\delta(i-j)$ initiated at time $j$ at the bottom ( $N$ th interface; not a free surface) of the medium results in a reflection response $r_{i, j}^{\prime}$ at the bottom and a transmission response $x_{i, j}^{\prime}$ at the top, which is promptly reflected back into the medium by the free surface at the top. We now argue

$$
x_{i, j}^{\prime}=x_{-j,-i} .
$$


To see this, consider the first non-zero values of the transmission responses. For probing from above starting at time $j=0$ we have

$$
x_{N / 2,0}=\prod_{i=1}^{N} \sqrt{1-k_{i}^{2}(i / 2)}
$$

and for probing from below starting at time $j=-N / 2$ we have

$$
x_{0,-N / 2}^{\prime}=\prod_{i=N}^{1} \sqrt{1-k_{i}^{2}(-i / 2)}=x_{N / 2,0}
$$

since $k_{n}(-i)=k_{n}(i)$ and there is no transmission at the free surface (0th interface). Some reflection shows that this principle holds in general.

\subsection{Green's function for time-varying layered medium}

Let $H^{n}(i, k)$ be the matrix Green's function from the waves at the surface $n=0$ at time $k$ to the waves just below the $n$th interface at time $i$, for an experiment initiated at time $j$. Then

$$
\begin{aligned}
& d_{n}(i, j)=\sum_{k}\left[H_{1,1}^{n}(i, k) d_{0}(k, j)+H_{1,2}^{n}(i, k) u_{0}(k, j)\right] \\
& u_{n}(i, j)=\sum_{k}\left[H_{2,1}^{n}(i, k) d_{0}(k, j)+H_{2,2}^{n}(i, k) u_{0}(k, j)\right] .
\end{aligned}
$$

$H^{n}(i, k)$ satisfies (2.1) without the factor $t_{n}(i)$ (we will reinsert this factor later; its absence does not affect the following development):

$$
\left[\begin{array}{ll}
H_{1,1}^{n}(i, k) & H_{1,2}^{n}(i, k) \\
H_{2,1}^{n}(i, k) & H_{2,2}^{n}(i, k)
\end{array}\right]=\left[\begin{array}{cc}
1 & -k_{n}(i) \\
-k_{n}(i) & 1
\end{array}\right]\left[\begin{array}{ll}
H_{1,1}^{n-1}\left(i-\frac{1}{2}, k\right) & H_{1,2}^{n-1}\left(i-\frac{1}{2}, k\right) \\
H_{2,1}^{n-1}\left(i+\frac{1}{2}, k\right) & H_{2,2}^{n-1}\left(i+\frac{1}{2}, k\right)
\end{array}\right]
$$

with the initial condition

$$
\left[\begin{array}{ll}
H_{1,1}^{0}(i, k) & H_{1,2}^{0}(i, k) \\
H_{2,1}^{0}(i, k) & H_{2,2}^{0}(i, k)
\end{array}\right]=\left[\begin{array}{cc}
\delta(i-k) & 0 \\
0 & \delta(i-k)
\end{array}\right] .
$$

Note that the time $j$ at which the probing impulse is at the surface $n=0$ does not appear in $H^{n}(i, k)$ since $H^{n}(i, k)$ is a property of the medium itself and thus is independent of any particular probing of it.

Now reverse time by replacing $i$ with $-i, j$ with $-j$ and $k$ with $-k$. This exchanges the downgoing and upgoing waves in each layer (recall we did not look at the internal waves in section 4.1). In fact, $d_{n}(-i,-j)=u_{n}(i, j)$ (to see this, reverse time in (2.1) and use the assumption $\left.k_{n}(i)=k_{n}(-i)\right)$. Then (4.10) becomes

$$
\begin{aligned}
& u_{n}(i, j)=\sum_{k}\left[H_{1,1}^{n}(-i,-k) u_{0}(k, j)+H_{1,2}^{n}(-i,-k) d_{0}(k, j)\right] \\
& d_{n}(i, j)=\sum_{k}\left[H_{2,1}^{n}(-i,-k) u_{0}(k, j)+H_{2,2}^{n}(-i,-k) d_{0}(k, j)\right] .
\end{aligned}
$$

Comparing (4.8) with (4.11) shows that the elements of $H^{n}(i, k)$ are related by

$$
H_{1,1}^{n}(-i,-k)=H_{2,2}^{n}(i, k) \quad H_{1,2}^{n}(-i,-k)=H_{2,1}^{n}(i, k)
$$

i.e. $H^{n}(i, k)$ has centrosymmetric structure under time reversal. Since $H^{n}(i, k)$ represents the effect of a succession of centrosymmetric layer matrices (2.1), this is no surprise.

Inserting (4.12) into (4.9) and postmultiplying by $[1,1]^{T}$ gives

$$
\left[\begin{array}{c}
\left(H_{1,1}^{n}+H_{1,2}^{n}\right)(i, k) \\
\left(H_{1,1}^{n}+H_{1,2}^{n}\right)(-i,-k)
\end{array}\right]=\left[\begin{array}{cc}
1 & -k_{n}(i) \\
-k_{n}(i) & 1
\end{array}\right]\left[\begin{array}{c}
\left(H_{1,1}^{n-1}+H_{1,2}^{n-1}\right)\left(i-\frac{1}{2}, k\right) \\
\left(H_{1,1}^{n-1}+H_{1,2}^{n-1}\right)\left(-i+\frac{1}{2},-k\right)
\end{array}\right]
$$


initialized at $n=0$ with $\delta(i-k)[1,1]^{T}$ (see (4.10); compare with (2.1)). An induction argument shows that $H_{1,1}^{n}(i, k)+H_{1,2}^{n}(i, k)$ has the form

$$
H_{1,1}^{n}(i, k)+H_{1,2}^{n}(i, k)= \begin{cases}0 & \text { if }|i-k|>n / 2 \\ -k_{n}(i) & \text { if } i-k=-n / 2\end{cases}
$$

so that we may obtain $k_{n}(i)$ from $H_{1,1}^{n}(i, k)+H_{1,2}^{n}(i, k)$.

\subsection{Nested non-Toeplitz systems of equations}

Inserting the boundary conditions (2.2) into (4.10) and using (4.12) gives

$$
\begin{aligned}
x_{i, j} & =\sum_{k}\left[H_{1,1}^{N}(i, k)\left(\delta(k-j)+r_{k, j}\right)+H_{1,2}^{N}(i, k) r_{k, j}\right] \\
& =\sum_{k}\left(H_{1,1}^{N}(i, k)+H_{1,2}^{N}(i, k)\right) r_{k, j}+H_{1,1}^{N}(i, j) \\
0= & \sum_{k}\left[H_{2,1}^{N}(i, k)\left(\delta(k-j)+r_{k, j}\right)+H_{2,2}^{N}(i, k) r_{k, j}\right] \\
= & \sum_{k}\left(H_{1,1}^{N}(i, k)+H_{1,2}^{N}(i, k)\right) r_{-k,-j}+H_{1,2}^{N}(i, j)
\end{aligned}
$$

where we have reversed time in (4.15b). Adding (4.15a) and (4.15b) gives

$$
\sum_{k}\left[\left(H_{1,1}^{N}(i, k)+H_{1,2}^{N}(i, k)\right)\left(\delta(k-j)+r_{k, j}+r_{-k,-j}\right)\right]=x_{i, j}
$$

Noting by causality that $x_{i, j}=0$ for $i<N / 2+j$ and $r_{k, j}=0$ for $k<j+1$, and using (4.1), we can write (4.16) as the system of equations

$$
\begin{array}{r}
{\left[\begin{array}{ccccc}
1 & r_{i-N / 2+1, i-N / 2} & r_{i-N / 2+2, i-N / 2} & \cdots & r_{i+N / 2, i-N / 2} \\
r_{i-N / 2+1, i-N / 2} & 1 & r_{i-N / 2+2, i-N / 2+1} & \cdots & r_{i+N / 2, i-N / 2+1} \\
r_{i-N / 2+2, i-N / 2} & r_{i-N / 2+2, i-N / 2+1} & 1 & \cdots & r_{i+N / 2, i-N / 2+2} \\
\vdots & \vdots & \vdots & \vdots & \vdots \\
r_{i+N / 2, i-N / 2} & r_{i+N / 2, i-N / 2+1} & r_{i+N / 2, i-N / 2+2} & \cdots & 1
\end{array}\right]} \\
\times\left[\begin{array}{c}
H_{1,1}^{N}(i, i-N / 2)+H_{1,2}^{N}(i, i-N / 2) \\
H_{1,1}^{N}(i, i-N / 2+1)+H_{1,2}^{N}(i, i-N / 2+1) \\
\vdots \\
H_{1,1}^{N}(i, i+N / 2-1)+H_{1,2}^{N}(i, i+N / 2-1) \\
H_{1,1}^{N}(i, i+N / 2)+H_{1,2}^{N}(i, i+N / 2)
\end{array}\right]=\left[\begin{array}{c}
x_{i, i-N / 2} \\
0 \\
\vdots \\
0 \\
0
\end{array}\right]
\end{array}
$$

where $x_{i, i-N / 2}=\prod_{k=1}^{N} t_{k}(i-N / 2+k / 2$ ) (compare with (4.6)). Note that only here does the transmission loss factor enter into the equations.

In (4.17) we can replace $N(=$ total number of layers) with any $n$ and (4.17) will still hold, since there will be no upgoing wave at depth $n$ by time causality. We can thus reconstruct the time-varying layered medium by solving (4.17) for $n=1,2 \ldots N$ where $N$ is the total number of layers; these are nested systems of equations. The time-varying reflection coefficients are found using (4.14).

\subsection{Feasibility condition}

Now return to the experiment in which the medium is probed from the bottom, as described by boundary conditions (4.4). The counterpart to (4.15), again derived by inserting boundary 
conditions (4.4) into (4.10) and using (4.12), is

$r_{i, j}^{\prime}=\sum_{k}\left[H_{1,1}^{N}(i, k) x_{k, j}^{\prime}+H_{1,2}^{N}(i, k) x_{k, j}^{\prime}\right]$

$\delta(i-j)=\sum_{k}\left[H_{2,1}^{N}(i, k) x_{k, j}^{\prime}+H_{2,2}^{N}(i, k) x_{k, j}^{\prime}\right]=\sum_{k}\left(H_{1,1}^{N}(i, k)+H_{1,2}^{N}(i, k)\right) x_{j, k}$

where we have again reversed time in (4.18b) and used (4.5). Equation (4.18b) can be written as a matrix equation $\left(H_{1,1}+H_{1,2}\right) X^{T}=I$, where the $(i, j)$ th element of each matrix is $H_{1,1}^{N}(i, j)+H_{1,2}^{N}(i, j)$ and $x_{i, j}$, and the former has non-zero elements only for $i-N / 2 \leqslant j \leqslant i+N / 2$ due to (4.14). Since a matrix and its inverse commute, we have

$$
\delta(i-k)=\sum_{m} x_{m, i}\left(H_{1,1}^{N}(m, k)+H_{1,2}^{N}(m, k)\right) .
$$

In (4.16) replace $i$ with $m$, multiply by $x_{m, i}$, sum over $m$, and use (4.1) and (4.19) to get the very important result

$$
\delta(i-j)+r_{i, j}+r_{j, i}=\sum_{m} x_{m, i} x_{m, j} .
$$

Equation (4.20) shows that the matrix in (4.17) is positive semidefinite, and its Cholesky factor is the matrix $X^{T}$ defined above. This is a generalization of the result of [7], in which it was shown that for a time-invariant medium the two-sided free surface reflection response is the autocorrelation of the transmission response.

The significance of (4.20) is that the reflection responses $r_{i, j}$ are such that the matrix in (4.17) is positive definite. If these responses are corrupted by noise to the point where this matrix is not positive definite, then none of the above methods work and indeed should not work since the data are infeasible, i.e. could not possibly have arisen from a medium described by (2.1). Such noisy data can easily be rendered feasible by projecting the matrix in (4.17) onto the space of positive definite matrices. One way to do this is to adjust negative eigenvalues to be slightly greater than zero; this is the minimum (in the Hilbert-Schmidt norm) noisy data perturbation required to produce feasible data.

\subsection{Comments}

(1) The layer-stripping algorithm of section 4.3 can now be interpreted as an algorithm for solving the nested non-Toeplitz systems of equations (4.17). However, since there is no structure to exploit (other than being nested), it is not a proper 'fast algorithm', and other methods for inverting nested matrices may be preferred. But the elegant physical interpretation of layer stripping still applies, and stability considerations also make it preferable.

(2) For the special case of a time-invariant medium $\left(k_{n}(i)\right.$ independent of time $\left.i\right)$, we see that $H^{n}(i, k)=H^{n}(i-k)$ and (4.8) becomes a convolution. This allows us to use the $z$-transform, and the development of sections 4.2 and 4.3 reduces to the well known one in [2,4]; all equations in sections 4.2 and 4.3 have their counterparts in [2] or [4]. We also have $r_{i, j}=r_{i-j}$, so that the system matrix in (4.17) becomes a Toeplitz matrix, which is solved (its Cholesky factorization is computed) using the Schur algorithm of section 3; (4.20) reduces to the result of Kunetz and d'Erceville [7].

(3) The material in section 4.1 seems to be new, even for time-invariant media. This provides an interesting physical interpretation of why a symmetric Toeplitz system of equations arises in [2,4]. Feasibility condition (4.20) also does not seem to have received sufficient attention, even in the time-invariant case; note the implications for using layerstripping algorithms. 
(4) Scale depth index $n$ and $k_{n}(i)$ by $\Delta$, and time index $i$ by $2 \Delta$ (this changes the wavespeed from two to unity) and let $\Delta \rightarrow 0$. The discrete medium described by (2.1) becomes a continuous medium described by

$$
\begin{aligned}
& \left(\frac{\partial}{\partial x}+\frac{\partial}{\partial t}\right) D(x, t)=-k(x, t) U(x, t) \\
& \left(\frac{\partial}{\partial x}-\frac{\partial}{\partial t}\right) U(x, t)=-k(x, t) D(x, t)
\end{aligned}
$$

and (4.16) and (4.17) become the integral equation

$$
r(x, t)+H(x, t)+\int_{-x}^{x} H(x, u) r(u, s) \mathrm{d} u=0 \quad-x<t<x
$$

where $r(x, t)$ is the free-surface reflection response at time $x$ to an impulse at time $t$, and $k(x, t)$ is the reflectivity function at depth $x$ at time $t$. For time-invariant media, $r(x, t)=r(x-t)$ and (4.22) becomes the Krein integral equation, so that (4.22) can be viewed as a generalized Krein integral equation.

\section{Applications}

\subsection{Reconstruction of rapidly curing layered media}

The most obvious application of the above results is the reconstruction of a medium whose physical parameters are actually changing rapidly with time. This arises in non-destructive testing of layered composites, in which some layers are quick setting adhesives bonding the layers on either side. If the curing time is of the order of milliseconds, then repeated testing under the assumption of a time-invariant medium will give incorrect results, since the medium is changing while a single test is in progress.

The probing impulses $\delta(i-j)$ for successive times $j$ can be produced from highfrequency vibrators. However, separating out the $r_{i, j}$ is a significant problem, since there is no way to distinguish $r_{4,0}, r_{4,1}$ and $r_{4,2}$ from each other (all are measured at the same time $i=4)$.

One way to solve this problem is to use different pulses at different times $j$. Note that the actual pulse shape need not be a true impulse-it need only have a support smaller than unity to prevent overlap with various reflections at nearby times. Since all reflections of a given pulse shape have the same shape as the original pulse, the pulses (and hence results of experiments at different times $j$ ) can be distinguished from each other if the pulses are orthogonal. That is, in the interval $4 \leqslant t \leqslant 5$ we observe $r_{4,0} \phi_{0}(t)+r_{4,1} \phi_{1}(t)+r_{4,2} \phi_{2}(t)$, where the $\phi_{j}(t)$ are orthogonal time-limited functions, so that $r_{4, j}$ can be found by multiplying by $\phi_{j}(t-4)$ and integrating.

Thus we need a sequence of pulses that are (1) orthogonal; (2) time-limited; and (3) easily generated in succession. This immediately suggests wavelet basis functions, since time scaling by powers of two produces orthogonal functions. In particular, the Haar functions would seem to be a good choice, due to their simplicity. If $\phi_{0}(t)$ is the basic Haar function

$$
\phi(t)= \begin{cases}1 & \text { if } 0<t<\frac{1}{2} \\ -1 & \text { if } \frac{1}{2}<t<1 \\ 0 & \text { if otherwise }\end{cases}
$$


then the layered medium could be probed with $\sum_{i=0}^{N} 2^{i} \phi\left(2^{i}(t-i)\right)$, the response $R(t)$ to this measured, and then

$$
r_{i, j}=\int_{t=i}^{i+1} R(t) 2^{j} \phi\left(2^{j} t\right) \mathrm{d} t .
$$

Daubechies wavelet basis functions could also be used, although these would be much harder to generate.

Another possibility is to use the lateral extent of the medium. Probing the medium with a spatially localized pulse at an angle of $\theta$ to the normal would produce (specular) reflected responses at an angle of $-\theta$ to the normal. By sweeping the pulser through different angles, pulses at different times would propagate through the medium at different angles $\theta$, and thus pulses from different experiments could be separated out by noting their angles. However, the vertical component of the velocity of propagation would be different for each pulse, so each copy of (3.3) in the layer-stripping algorithm would have a different propagation speed. By choosing $\theta_{m}=\cos ^{-1}(1 / m)$ for various positive integers $m, k_{n}(i)$ will still be computed at any time $i$ from some copy of (3.3); this will then be broadcasted to all other copies of (3.3). Note that infinite-extent non-normal-incidence impulsive plane waves cannot be used.

\subsection{Time-varying discrete Miura transform and the Schrödinger equation}

We now consider the inverse scattering problem for a medium not described by the discrete wavesystem (2.1) but by the following equation, which we will call a discrete Schrödinger equation with a time-varying potential:

$h_{n+1}(i, j)+h_{n-1}(i, j)-h_{n}\left(i-\frac{1}{2}, j\right)-h_{n}\left(i+\frac{1}{2}, j\right)=V_{n}(i) h_{n-1}(i, j)$.

Scale depth index $n$ by $\Delta$, time index $i$ by $2 \Delta$ (this changes the wavespeed from two to unity) and $V_{n}(i)$ by $\Delta^{2}$, and let $\Delta \rightarrow 0$. The discrete medium described by (5.3) becomes a continuous medium described by

$$
\left(\frac{\partial^{2}}{\partial x^{2}}-\frac{\partial^{2}}{\partial t^{2}}\right) h(x, t)=V(x, t) h(x, t) .
$$

If $V(x, t)$ is independent of time, (5.4) is the inverse Fourier transform of a Schrödinger equation. Allowing the potential $V(x, t)$ to vary with time means that (5.4) is not just the inverse Fourier transform of a Schrödinger equation, but the time-domain interpretation of a wave operator on the left-hand side and a now time-varying scattering potential on the right-hand side still holds, so we refer to (5.3) and (5.4) as Schrödinger equations for convenience.

Suppose for the moment that the discrete potential $V_{n}(i)$ in (5.3) does not vary with time $i$. Then the discrete wavesystem (2.1) and the discrete Schrödinger equation (5.3) are related by the discrete Miura transform [8]

$$
V_{n-1}=k_{n} k_{n-1}+\left(k_{n}-k_{n-1}\right) \quad h_{n-1}(i, j)=\left(d_{n}\left(i-\frac{1}{2}, j\right)+u_{n}\left(i+\frac{1}{2}, j\right)\right) t_{n-1} .
$$

The continuous limit as $\Delta \rightarrow 0$ of (5.5) is

$$
V(x)=k(x)^{2}+\mathrm{d} k(x) / \mathrm{d} x \quad h(x, t)=d(x, t)+u(x, t)
$$

which is the (continuous) Miura transform introduced in [3]; the first equation of (5.6) arises in soliton theory and the inverse scattering transform. The physical interpretation of this transform is clear: instead of propagating downgoing and upgoing waves, we are propagating the field quantities (e.g. voltage, current, pressure, displacement) from which 
these waves are defined. At each depth we split the field quantity up into the sum of downgoing and upgoing waves, which propagate by the first-difference (derivative) operator in $(2.1)((4.21))$, rather than the second-difference (derivative) operator in $(5.3)((5.4))$. The relation between (2.1) and (5.3) using (5.5) (and between (4.21) and (5.4) using (5.6)) can be verified algebraically for time-invariant media.

Note from (5.5) that each interface produces two non-zero values of the discrete potential $V_{n}$ but only one non-zero value of $k_{n}$ (see (2.1)). Now recall that throughout this work we assume that the medium changes in time while waves are between interfaces, so that we need not consider what happens when an interface changes as a wave is passing through it. In order to maintain this assumption, we now need to assume that only even-indexed interfaces have non-zero $k_{n}(i)$, so that field quantities propagating according to (5.3) are not affected by a change in the medium as they propagate through an interface. That is, we need 'guard' bands around each interface so that the propagating field quantities are not affected at the moments when the medium, and thus the $k_{n}(i)$ and $V_{n}(i)$, change with time. These guard bands simply mean that the layers have to be twice as thick as before. Alternatively, we may discretize twice as finely (halve $\Delta$ ) for the field quantity indices (and wave operator) as for the $V_{n}(i)$ indices, which produces the same effect for a discretized medium.

With this assumption the discrete Miura transform (5.5) generalizes directly to

$$
\begin{aligned}
& V_{n-1}(i)=k_{n}(i) k_{n-1}(i)+\left(k_{n}(i)-k_{n-1}(i)\right) \\
& h_{n-1}(i, j)=\left(d_{n}\left(i-\frac{1}{2}, j\right)+u_{n}\left(i+\frac{1}{2}, j\right)\right) t_{n-1}
\end{aligned}
$$

(note that one of $k_{n}(i)$ and $k_{n-1}(i)$ will be zero). That is, as the interface reflection coefficients $k_{n}(i)$ change with time, the discrete potentials $V_{n}(i)$ will also change with time, but will do so at times when the propagating field quantities are not only between interfaces, but between the effects of interfaces on $V_{n}(i)$. Thus there will be no effects due to changes in the medium as a field quantity propagates through an interface.

\subsection{The two-dimensional inverse resistivity problem}

The $2 \mathrm{D}$ inverse resistivity problem of reconstructing the $2 \mathrm{D}$ resistivity (ohms) $\rho(x, y)$ of a 2D medium from surface measurements of the potential (voltage) response to an injected current can be formulated as a time-varying $1 \mathrm{D}$ inverse scattering problem. This problem has attracted a great interest recently [5,6]. For more details and references see [13], or any recent IEEE Engineering in Medicine and Biology conference proceedings.

Consider a 2D dielectric medium with resistivity (reciprocal of conductivity) $\rho(x, y)$. Direct current is introduced into the medium at point $\left(x=0, y_{0}\right)$ through an electrode, and the resulting electrical potential $v(x=0, y)$ induced at the surface $x=0$ is measured. We assume that the surface is a perfect insulator, so that the vertical component of the current at the surface away from the source is zero, and that $\rho(x, y)$ is constant outside some compact region. The aim is to reconstruct $\rho(x, y)$ from the measured electrical potential $v(x=0, y)$.

The basic equations for the 2D inverse resistivity problem are Ohm's law and Kirchhoff's current law $[5,6,9]$

$$
\nabla v(x, y)=\rho(x, y) \boldsymbol{j}(x, y) \quad \nabla \cdot \boldsymbol{j}(x, y)=0
$$

where $\boldsymbol{j}(x, y)$ is the current density. The boundary conditions are $v(x=0, y), \boldsymbol{j}_{z}(x=$ $0, y \neq 0)=0$, and $j(x, y) \rightarrow 0$ as $\left(x^{2}+y^{2}\right) \rightarrow \infty$. The normalized electrical potential $h(x, y)=v(x, y) / \sqrt{\rho(x, y)}$ satisfies [9]

$$
\nabla^{2} h(x, y)=V(x, y) h(x, y) \quad V=\rho^{1 / 2} \nabla^{2} \rho^{-1 / 2}
$$


so that if the medium is locally homogeneous then $V(x, y)=0$ and the normalized electrical potential properly satisfies Laplace's equation.

We now use a transformation that maps solutions of the elliptic equation (5.9) into solutions of the hyperbolic equation (5.4). This transformation, used previously in [9-12], consists of Fourier transforming $h(x, y)$ and $V(x, y)$ from $y$ to $k$, performing an analytic continuation into the entire right half of the complex $k$-plane from values on the positive real axis and then performing an inverse Laplace transform from $k$ to $t$. That is,

$$
h^{\prime}(x, t)=\mathcal{L}_{i k \rightarrow t}^{-1}\left\{k \mathcal{F}_{y \rightarrow k}\{h(x, y)\}\right\}
$$

with $V^{\prime}(x, t)$ defined similarly. It is clear that $h^{\prime}(x, t)$ and $V^{\prime}(x, t)$ satisfy (5.4), with the known boundary condition $h^{\prime}(x=0, t)$ causal in $t$ (due to the inverse Laplace transform). The extra $k$ is necessary to produce an impulse $\delta(t)$ in the boundary condition for (5.4). This transformation has been discussed in detail in [9-12]; we do not repeat all of the details here.

At this point the 2D inverse resistivity problem has been transformed into a $1 \mathrm{D}$ timevarying inverse scattering problem described by (5.4), which can be discretized into (5.3), which in turn can be related to (2.1) through the Miura transform (5.5). This has an important implication about data for the $2 \mathrm{D}$ inverse resistivity problem: the data transformed using (5.10) must satisfy the feasibility condition described in section 4.4. If the data are corrupted by noise that makes the transformed data infeasible (and even a small amount of noise can do this), then neither layer stripping nor any other exact procedure will work, as discussed in section 4.4. The correction procedure proposed in section 4 could be applied to these noisy infeasible data to render them feasible. This feasiblity condition seems to be a new result for $2 \mathrm{D}$ inverse resistivity.

We have quickly sketched the application of time-varying 1D inverse scattering results to the $2 \mathrm{D}$ inverse resistivity problem defined on a half-space. For a detailed treatment of the relation between time-varying $1 \mathrm{D}$ inverse scattering and the 2D impedance tomography problem see [14].

\section{An illustrative numerical example}

We present an illustrative numerical example that demonstrates the algorithms and the data sorting procedure. It also demonstrates the necessity of impulse reflection response data from impulses at different times, since it explicitly demonstrates the non-uniqueness of the time-varying inverse scattering problem without these data. The example is chosen to be simple enough so that the reader can actually understand how the equations work and what they are doing, and why the answers are what they are. We believe this is more useful than a simple numerical verification.

\subsection{Example problem}

A time-varying 1D layered medium with a free (perfectly reflecting) surface is probed using an impulse $\delta(i)$ just below the free surface, as was done in $[1-4,7,8]$ and as discussed in section 2.2. The reflection response is found to be $r_{i, 0}=(0.9)^{i^{2}}, i>0$. What are the reflection coefficients?

In the absence of any other information we might conclude that the reflection coefficients are $k_{n}(i)=-(-0.9)^{n}$, since it is well known in the signal processing literature that the reflection coefficients of the lattice filter associated with covariance $(\rho)^{i^{2}}$ are $k_{n}(i)=$ $-(-\rho)^{n}$ (see [15] for references and an inverse scattering interpretation of this fact; note 
the sign change in $k_{n}$ ). The first two values of $r_{i, 0}$ would be $r_{1,0}=k_{1}=0.9$ and $r_{2,0}=k_{2}\left(1-k_{1}^{2}\right)+k_{1}^{2}=-0.81\left(1-(0.9)^{2}\right)+(0.9)^{2}=(0.9)^{4}$ (note the two-way transmission loss factor).

However, if the medium is probed using an impulse $\delta(i-j)$ at various times $j$ instead of just at time $j=0$, the reflection response is found to be

$$
r_{i, j}=(0.9)^{i^{2}-j^{2}} \quad i>j .
$$

Since $r_{i, j} \neq r_{i-j, 0}$ the medium is time varying, as discussed in section 4.5. The procedures proposed in this paper must be used to reconstruct $k_{n}(i)$.

\subsection{Example problem solution using layer stripping algorithm}

Implementing the algorithm of section 3 gives the following:

(1) Setting $n=1$ in (3.2) and using (2.2) gives

$$
k_{1}\left(\frac{1}{2}+j\right)=\frac{u_{0}(j+1, j)}{d_{0}(j, j)}=\frac{r_{j+1, j}}{1}=(0.9)^{\left[(j+1)^{2}-j^{2}\right]}=(0.9)^{(2 j+1)}
$$

which becomes $k_{1}(j)=(0.9)^{2 j}$. This is physically clear- $k_{1}(j)$ is found directly from the reflection response $r_{j+1, j}$.

(2) Setting $n=1$ in (2.1) and using (2.2) gives

$$
\left[\begin{array}{l}
d_{1}(i, j) \\
u_{1}(i, j)
\end{array}\right]=\left[\begin{array}{cc}
1 & -(0.9)^{2 i} \\
-(0.9)^{2 i} & 1
\end{array}\right]\left[\begin{array}{c}
(0.9)^{\left(i-\frac{1}{2}\right)^{2}-j^{2}} \\
(0.9)^{\left(i+\frac{1}{2}\right)^{2}-j^{2}}
\end{array}\right]=\left[\begin{array}{c}
(0.9)^{\left(i-\frac{1}{2}\right)^{2}-j^{2}}\left(1-(0.9)^{4 i}\right) \\
0
\end{array}\right]
$$

(3) Since $u_{1}(i, j)=0$ for all $i, j$, we know there are no more layers.

If there were any deeper layers, they would create an upgoing wave (a non-zero $u_{1}(i, j)$ ) at some time. Hence, this is now complete.

(4) The final answer is: $k_{1}(i)=(0.9)^{2 i} ; k_{n}(i)=0$ for $n>1$.

The physical picture is clear. The reflection response $r_{i, j}$ is caused by reverberations between the two layers of the medium (the free surface and $\left.k_{1}(i)=(0.9)^{2 i}\right)$. For a probing impulse $\delta(i)$ at time $j=0$, the first response is $r_{1,0}=0.9$ since the reflection occurs at time $i=\frac{1}{2}$. The second response is $r_{2,0}=0.9^{4}$ since the reflections occur at times $i=\frac{1}{2}$ and $i=3 / 2$ and $k_{1}\left(\frac{1}{2}\right) k_{1}\left(\frac{3}{2}\right)=(0.9)^{(1+3)}$. The third response is

$$
r_{3,0}=k_{1}\left(\frac{1}{2}\right) k_{1}\left(\frac{3}{2}\right) k_{1}\left(\frac{5}{2}\right)=(0.9)^{(1+3+5)}=(0.9)^{3^{2}} .
$$

It is clear that the quadratic exponent in $r_{i, 0}$ comes from the sum of the series $1+3+5+$ $\cdots+(2 n-1)=n^{2}$.

Note that even a simple two-layer time-varying medium can have a surprisingly complicated reflection response. More complex examples are too difficult to interpret physically.

\subsection{Example problem solution using systems of equations}

We can show explicitly the solution of the nested non-Toeplitz systems (4.17):

(1) setting $n=1$ and $i=\frac{1}{2}$ in (4.17) gives

$$
\left[\begin{array}{cc}
1 & r_{1,0} \\
r_{1,0} & 1
\end{array}\right]\left[\begin{array}{l}
H_{1,1}^{1}\left(\frac{1}{2}, 0\right)+H_{1,2}^{1}\left(\frac{1}{2}, 0\right) \\
H_{1,1}^{1}\left(\frac{1}{2}, 1\right)+H_{1,2}^{1}\left(\frac{1}{2}, 1\right)
\end{array}\right]=\left[\begin{array}{c}
x_{1 / 2,0} \\
0
\end{array}\right] .
$$


Inserting values and solving this linear system of equations gives

$$
\left[\begin{array}{cc}
1 & 0.9 \\
0.9 & 1
\end{array}\right]\left[\begin{array}{c}
1 \\
-0.9
\end{array}\right]=\left[\begin{array}{c}
0.19 \\
0
\end{array}\right] \text {. }
$$

Using (6.6) and (4.14) we have $k_{1}\left(\frac{1}{2}\right)=0.9$, as expected.

(2) Setting $n=1$ and $i=\frac{3}{2}$ in (4.17) gives

$$
\left[\begin{array}{cc}
1 & r_{2,1} \\
r_{2,1} & 1
\end{array}\right]\left[\begin{array}{c}
H_{1,1}^{1}\left(\frac{3}{2}, 1\right)+H_{1,2}^{1}\left(\frac{3}{2}, 1\right) \\
H_{1,1}^{1}\left(\frac{3}{2}, 2\right)+H_{1,2}^{1}(3 / 2,2)
\end{array}\right]=\left[\begin{array}{c}
x_{3 / 2,1} \\
0
\end{array}\right] .
$$

Inserting values and solving this linear system of equations gives

$$
\left[\begin{array}{cc}
1 & 0.729 \\
0.729 & 1
\end{array}\right]\left[\begin{array}{c}
1 \\
-0.729
\end{array}\right]=\left[\begin{array}{c}
0.469 \\
0
\end{array}\right] \text {. }
$$

Using (6.8) and (4.14) we have $k_{1}\left(\frac{3}{2}\right)=0.729=(0.9)^{3}$, as expected.

Other $k_{1}(i)$ can be found similarly.

(3) Setting $n=2$ and $i=1$ in (4.17) gives

$$
\left[\begin{array}{ccc}
1 & r_{1,0} & r_{2,0} \\
r_{1,0} & 1 & r_{2,1} \\
r_{2,0} & r_{2,1} & 1
\end{array}\right]\left[\begin{array}{l}
H_{1,1}^{2}(1,0)+H_{1,2}^{2}(1,0) \\
H_{1,1}^{2}(1,1)+H_{1,2}^{2}(1,1) \\
H_{1,1}^{2}(1,2)+H_{1,2}^{2}(1,2)
\end{array}\right]=\left[\begin{array}{c}
x_{1,0} \\
0 \\
0
\end{array}\right] .
$$

Inserting values and solving this linear system of equations gives

$$
\left[\begin{array}{ccc}
1 & 0.9 & 0.6561 \\
0.9 & 1 & 0.729 \\
0.6561 & 0.729 & 1
\end{array}\right]\left[\begin{array}{c}
1 \\
-0.9 \\
0
\end{array}\right]=\left[\begin{array}{c}
0.19 \\
0 \\
0
\end{array}\right]
$$

Using (6.10) and (4.14) we have $k_{2}(1)=0$, as expected.

Other $k_{2}(i)$ can be shown to be zero similarly.

This simple example shows how the solution of the nested non-Toeplitz systems of equations can also be used to reconstruct the medium. The advantages of using the layerstripping approach should be obvious.

\section{Conclusion}

We have formulated and presented algorithms and applications for the inverse scattering problem for a time-varying 1D layered medium. The problem can be solved either by solving a series of nested systems of equations or by propagating a set of coupled layerstripping algorithms with reflection coefficients varying in time. Our results reduced to previous results for time-invariant media. We also presented a time-varying discrete Miura transform linking the time-varying discrete wavesystem inverse scattering problem to a timevarying discrete Schrödinger equation, which in turn is related to the 2D inverse resistivity problem by an inverse Laplace-Fourier transformation. We showed that the data matrix of reflection data from experiments initiating at different times must be positive definite; this feasibility result also applies to the 2D inverse resistivity problem (after transforming the data). Exploring the implications of the latter result, especially with regards to rendering noisy infeasible data feasible, is the obvious next step in this research. We have formulated the problem in more detail in [14].

\section{Acknowledgments}

The author would like to thank the reviewers for some helpful comments. This research was supported by the Office of Naval Research under grant no N00014-94-1-0519. 


\section{References}

[1] Bube K P and Burridge R 1983 The one-dimensional inverse problem of reflection seismology SIAM Rev. 25 497-559

[2] Bruckstein A M and Kailath T 1987 Inverse scattering for discrete transmission-line models SIAM Rev. 29 359-89

[3] Bruckstein A M, Levy B C and Kailath T 1985 Differential methods in inverse scattering SIAM J. Appl. Math. 45 312-35

[4] Ware J A and Aki K 1969 Continuous and discrete inverse scattering problems in a stratified elastic medium I. Plane waves at normal incidence J. Acoust. Soc. Am. 45 911-21

[5] Isaacson D and Cheney M 1990 Current problems in impedance imaging Inverse Problems in Partial Differential equations ed D Colton, R Ewing and W Rundell (Philadelphia, PA: SIAM)

[6] Cheney M, Isaacson D, Isaacson E L and Somersalo E 1991 A layer-stripping approach to impedance imaging 7th Annual Review of Progress in Applied Computational Electromagnetics (Naval Postgraduate School, March)

[7] Kunetz G and d'Erceville I 1962 Sur certaine proprieties d'une onde acoustique plane de compression dans un milieu stratifie Ann. Geophys. 18 351-9

[8] Yagle A E 1989 Fast algorithms for estimation and signal processing: an inverse scattering framework IEEE Trans. Acoust. Speech Sig. Process. 37 957-9

[9] Weidelt P 1972 The inverse problem of geomagnetic induction Z. Geophys. 38 257-89

[10] Coen S and M. W.-H. Yu M W-H 1981 The inverse problem of the direct current conductivity profile of a layered earth Geophysics 46 1702-13

[11] Levy B C 1985 Layer by layer reconstruction methods for the earth resistivity from direct current measurements IEEE Trans. Geosci. Rem. Sensing GE-23 841-50

[12] Yagle A E 1987 A layer stripping fast algorithm for the two-dimensional direct current inverse resistivity problem IEEE Trans. Geosci. Rem. Sensing GE-25 558-63

[13] Yorkey T J, Webster J G and Tompkins W J 1987 Comparing reconstruction methods for electrical impedance tomography IEEE Trans. Biomed. Eng. BME-34 843-52

[14] Yagle A E Discrete layer stripping and feasibility conditions for 2D impedance tomography in polar coordinates Inverse Problems revision submitted

[15] A.E. Yagle 1988 On geometric sequences of reflection coefficients and Gaussian autocorrelations Proc. IEEE 76 1372-4 